\title{
A Refutation Procedure for Proving Satisfiability of Constraint Specifications on XML Documents $\rrbracket^{*}$
}

\author{
Marisa Navarro ${ }^{1}$ and Fernando Orejas ${ }^{2}$ \\ 1 Universidad del País Vasco (UPV/EHU), San Sebastián, Spain \\ marisa.navarro@ehu.es \\ 2 Universitat Politècnica de Catalunya, Barcelona, Spain \\ orejas@lsi.upc.edu
}

\begin{abstract}
In this paper we first present three sorts of constraints (positive, negative and conditional ones) to specify that certain patterns must be satisfied in an XML document. These constraints are built on boolean XPath patterns. We define a specification as a set of clauses whose literals are constraints. Then, to reason about these specifications, we study some sound rules which permit to infer, subsume or simplify clauses. The main goal is to design a refutation procedure (based on these rules) to test if a given specification is satisfiable or not. We have formally proven the soundness of our procedure and we study the completeness and termination of the proposed method.
\end{abstract}

\section{Introduction}

$\mathrm{XPath}[12,16]$ is a well-known language for navigating an XML document (or XML tree) and returning a set of answer nodes. Since XPath is used in many XML query languages as XQuery, XSLT or XML Schema among others [15, 13, 14], a great amount of papers deal with different aspects on different fragments of XPath. For instance, in [3] an overview of formal results on XPath is presented concerning the expressiveness of several fragments, complexity bounds for evaluation of XPath queries, as well as static analysis of XPath queries. In [4] they study the problem of determining, given a query $p$ (in a given XPath fragment) and a DTD D, whether there exists an XML document conforming to D and satisfying p. They show that the complexity ranges from PTIME to undecidable, depending on the XPath fragment and the DTD chosen. The work presented in [5] deals with the same problem (in a particular case) and it uses Hybrid Modal Logic to model the documents and some class of schemas and constraints. They provide a tableau proof technique for constraint satisfiability testing in the presence of schemas.

Our approach is different than the previous ones in two aspects. On the one hand, we do not consider any DTD or schema, and we use a simple fragment of XPath. In this sense our approach is simpler than previous ones. But, on the other hand, our aim is to define specifications of classes of XML documents as sets of constraints on these documents, and to provide a form of reasoning about these specifications. In this sense, our main question is satisfiability, that is, given a set of constraints $\mathrm{S}$, whether there exists an XML document satisfying all constraints in S. Moreover, we are looking for refutation procedures, based on sound and complete inference rules. In addition to checking satisfiability, these rules can be used to infer other constraints from the given set, which can help us to optimize the given specification.

Some other work, which shares part of our aims, is the approach for the specification and verification of semi-structured documents based on extending a fragment of first-order logic [2, 7]. They present specification languages that allow us to specify classes of documents, and tools that allow us to check if a given document (or a set of documents) follows a given specification. However, they do not consider the problem of defining deductive tools to analyze specifications, for instance to look for inconsistencies.

${ }^{*}$ This work has been partially supported by the Spanish Project TIN2013-46181-C2-2-R, the Basque Project GIU12/26, and grant UFI11/45.

T. Kutsia, A. Voronkov (eds.), SCSS 2014 (EPiC Series, vol. 30), pp. 4761 


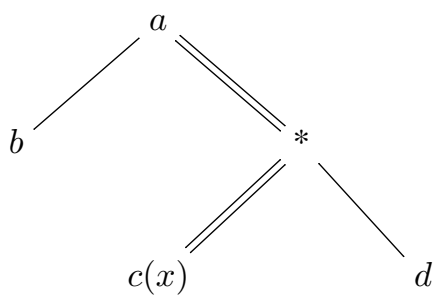

Figure 1: A tree pattern with answer node (marked $x$ )

Schematron [8] has a more practical nature. It is a language and a tool that is part of an ISO standard (DSDL: Document Schema Description Languages). The language allows us to specify constraints on XML documents by describing directly XML patterns (using XML) and expressing properties about these patterns. Then, the tool allows us to check if a given XML document satisfies these constraints. However, as in the previous approach, Schematron provides no deductive capabilities. Finally, we consider the work presented in [10]. It shows how to use graph constraints as a specification formalism, which can be used to specify classes of semi-structured documents, and how to reason about these specifications, providing refutation procedures based on inference rules that are sound and complete.

We follow the main ideas given in [10] trying to apply them to XML documents. Our constraints are based on Xpath queries, as given in [9], which contain branching, label wildcards and can express descendant relationships between nodes. In particular, their tree patterns are an alternative representation of XPath queries consisting of node tests, the child axis (/), the descendant axis (//) and wildcards (*). The answer nodes are marked with $(x)$. For instance, Figure 1 shows a tree pattern $p$ that when applied to a given XML document $t$ (which is also represented by a tree but in this case without descendant axis or wildcards), it must check if the root node in $t$ is labelled $a$, if some child node of the root node in $t$ is labelled $b$, and if some descendant node of the root node in $t$ has both a child node labelled $d$ and a descendant node labelled $c$. If all of these conditions are satisfied, the application $p(t)$ will return a set with such last descendant nodes (the nodes marked with $x$ ); otherwise, it will return the empty set.

Since our purpose is to reason on XML documents by means of a set of constraints, and not to obtain the answer nodes, we shall consider tree patterns without answer marks (which are called Boolean tree patterns in [9]). The application of such a pattern to a document $t$ will return true, if $t$ satisfies the conditions specified by the pattern, or false otherwise.

We consider three kinds of (atomic) constraints. The first one is $\exists p$ where $p$ is a tree pattern. This constraint will be satisfied by a document $t$ if $p(t)$ is true. The second one is $\neg \exists p$ that will be satisfied by a document $t$ if $p(t)$ is false. The third sort of constraint is written $\forall(c: p \rightarrow q)$, where both $p$ and $q$ are tree patterns (related by $c$ in a special way) and, roughly speaking, it will be satisfied by a document $t$ if $p(t)$ implies $q(t)$. Nevertheless, the application of the ideas in [10] to our setting is not trivial, as discussed in Section 3

Our aim is to study adequate inference rules to find a sound and complete refutation procedure for checking satisfiability of a given specification. The inference rules take a format similar to the inference rules given in [10], but again the particularization to our setting needs to define appropriate operators and to prove new results. Moreover, these rules allow to infer, subsume and simplify clauses; that is, they permit to manipulate a specification and obtain another optimized specification which is semantically equivalent to the given one.

The paper is organized as follows. Section 2 contains some basic notions and notational conventions we are going to use along the paper. Section 3 introduces the three sorts of constraints that we use as literals of the clauses in a specification. Section 4 presents the main inference rules for our refutation 
procedure, proving soundness. We also give an example of refutation for a given specification. Then, in Section 5 we show that some other rules (named unfolding rules) are necessary in order to obtain completeness. We also add some subsumption and simplification rules to produce a more efficient procedure and we study the conditions for the termination of the refutation procedure. Having done this study, we propose in Section 6 a refutation procedure by defining how to apply the rules. Finally, in Section 7 we provide some conclusions and further work.

\section{Basic Definitions and Notations}

In this section we introduce some basic concepts and notations that are used along the paper.

\subsection{XML Documents and Patterns}

We consider an XML document as an unordered and unranked tree with nodes labelled from an infinite alphabet $\Sigma$. The symbols in $\Sigma$ can represent the element labels, attribute labels, and text values that can occur in documents. A document on $\Sigma=\{a, b, c, d, e, f, g\}$ is given in the right part of Figure 2 . By considering that the trees are unordered and unranked, the subtrees can commute (the "sibling ordering" is irrelevant), and there are no restrictions on the number of children a node can have.

Definition 2.1. Given a signature $\Sigma$, a document on $\Sigma$ is a tree $t$ whose nodes are labelled with symbols from $\Sigma$ and with one sort of edges denoted $/ . T_{\Sigma}$ denotes the set of all documents on alphabet $\Sigma$. Given a document $t \in T_{\Sigma}$, Nodes $(t)$ and Edges $(t)$ denote respectively the sets of nodes and edges in $t$; Root $(t)$ denotes its root node; and for each $n \in \operatorname{Nodes}(t)$, Label $(n)$ denotes the label of such a node $n$. Edges ${ }^{+}(t)$ denotes the transitive closure of Edges $(t)$. Each edge in Edge $(t)$ is represented $(x, y)$ with $x, y \in \operatorname{Nodes}(t)$. Each $(x, y) \in$ Edges $^{+}(t)$ represents a path in $t$ from node $x$ to node $y$.

As said above, we use patterns as an alternative representation of queries. In particular, we are interested in boolean patterns. A pattern on $\Sigma=\{a, b, c, d, e, f, g\}$ is given in the left part of Figure 2

Definition 2.2. Given a signature $\Sigma$, a pattern on $\Sigma$ is a tree $p$ whose nodes are labelled with symbols from $\Sigma \cup\{*\}$ and with two sorts of edges: the descendant edges denoted // and the child edges denoted/. $P_{\Sigma}$ denotes the set of all patterns on alphabet $\Sigma$. Given a pattern $p \in P_{\Sigma}$, we use the same notations as before: Nodes $(p)$, Edges $(p)$ and Root $(p)$ for the nodes, the edges and the root of $p$, and Label $(n)$ for

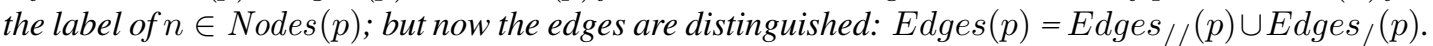
Edges $^{+}(p)$ denotes the transitive closure of Edges $(p)$, that is, $(x, y) \in$ Edges $^{+}(p)$ represents a path in $p$ from node $x$ to node $y$ with edges of type / or // along the path.

For the sake of simplicity, from now on we omit the signature $\Sigma$. Along this paper, patterns will be drawn in the figures as trees, but to write them textually we will use the following format: A pattern $p$ with root labelled $a$ and subtrees $p_{1}, \ldots, p_{n}$ will be textually written $p=a\left(! p_{1}\right) \ldots\left(! p_{n}\right)$ where each $p_{i}$ is recursively written in the same format, and ! being / or // to indicate the edge from the root to each subtree $p_{i}$. Some parenthesis can be omitted in the case of having only one subtree.

From the previous definitions, it is clear that documents coincide with a special case of patterns: those patterns with no label $*$ and with no edge of type //. For this reason, in the next section we will define the relation between two patterns, and as a particular case we will have defined the relation between a pattern and a document. 


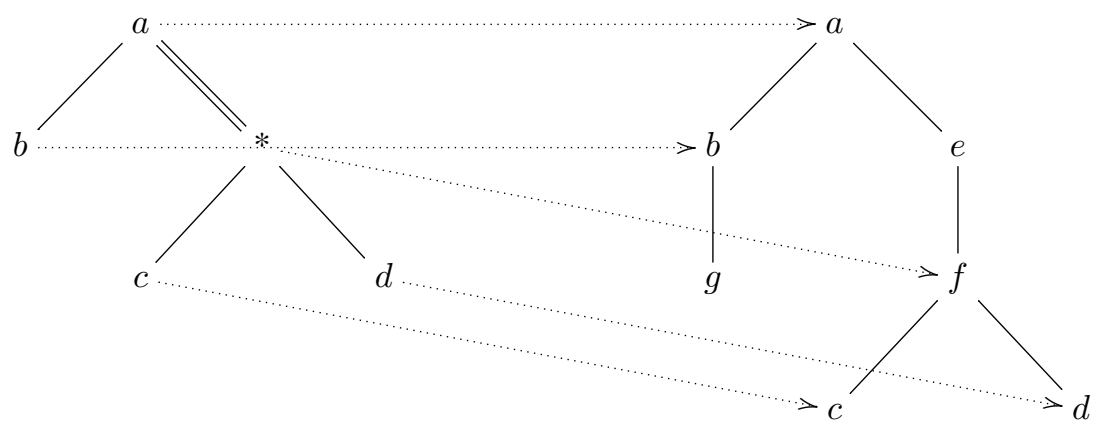

Figure 2: A pattern $p$, a document $t$ and a monomorphism $h: p \rightarrow t$

\subsection{Pattern Homomorphisms and Pattern Models}

We define here the notion of homomorphism between two patterns and also (as a particular case) the notion of homomorphism between a pattern and a document. The later one will be used to define which documents are the models of a pattern. That is, from a logical point of view, we can see patterns as formulae, documents as structures and we can define a notion of pattern satisfaction.

Definition 2.3. Given two patterns $p, q \in P$, a homomorphism from $p$ to $q$ is a function $h: \operatorname{Nodes}(p) \rightarrow$ Nodes $(q)$ satisfying the following conditions:

- Root-preserving: $h(\operatorname{Root}(p))=\operatorname{Root}(q)$;

- Label-preserving: For each $n \in(p)$, Label $(n)=*$ or Label $(n)=\operatorname{Label}(h(n))$;

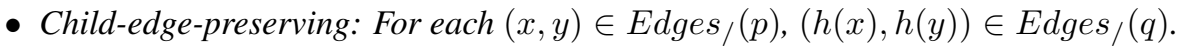

- Descendant-edge-preserving: For each $(x, y) \in \operatorname{Edges}_{/ /}(p),(h(x), h(y)) \in \operatorname{Edges}^{+}(q)$.

From now on, we will simply write $h: p \rightarrow q$ for $h: \operatorname{Nodes}(p) \rightarrow \operatorname{Nodes}(q)$. The definition of a homomorphism from a pattern $p$ to a document $t$ is the previous definition. Note that in this particular

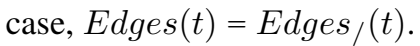

Definition 2.4. Given a pattern $p \in P$ and a document $t \in T$, we say that $\mathrm{t}$ satisfies $\mathrm{p}$, denoted $t \vDash p$, if there exists a monomorphism (i.e., an injective homomorphism) from $p$ to $t$. The model set of a pattern $p$ is the set of documents satisfying $p: \operatorname{Mod}(p)=\{t \in T \mid t \vDash p\}$

In Figure 2 there is an example of an injective homomorphism $h: p \rightarrow t$ from the pattern $p=$ $a(/ b)(/ / *(/ c)(/ d))$ to the document $t=a(/ e / f(/ c)(/ d))(/ b / g)$. The homomorphism $h$ is drawn with dotted arrows. We can see that the document $t$ satisfies the pattern $p$ because its root is labelled with $a$, it has a child node labelled $b$, and it has a descendant node (in the example labelled with $f$ ) with two child nodes labelled with $c$ and $d$ respectively. In Figure 3 there is an example of a monomorphism $h: p \rightarrow q$ from the pattern $p=* / / e$ to the pattern $q=a(/ e)(/ / b / c)$. The monomorphism $h$ is drawn with dotted arrows. The existence of such monomorphism implies that all models of $q$ are also models of $p$.

The following lemma relates monomorphisms and models for two patterns.

Lemma 2.1. Given two patterns $p, q \in P$ :

- If there exists a monomorphism $h: p \rightarrow q$ then $\operatorname{Mod}(q) \subseteq \operatorname{Mod}(p)$.

- $\operatorname{Mod}(q) \subseteq \operatorname{Mod}(p)$ does not imply that there is a monomorphism $h: p \rightarrow q$. 
Proof. The first point: Let $t$ be a document in $\operatorname{Mod}(q)$, then there exists a monomorphism $f$ from $q$ to $t$. Then the composition $f \circ h$ is a monomorphism from $p$ to $t$ and therefore the document $t$ is also a model for $p$. The second point is illustrated with an example in [9] .

\section{Constraints, Clauses and Specifications}

We take from [10] the notion of graph constraint to define our notion of pattern constraint. In that paper one sort of constraints is of the form $\exists C$, with $C$ being a graph. Then a given graph $G$ is defined to verify this constraint when $G$ contains $C$ as a subgraph. However, translating the ideas in [10] to our setting is not trivial mainly for two reasons. We deal with patterns that are trees having edges of type //, but the related notion of "path" is not considered for graph constraints. On the other hand, in the setting of graph constraints, models and formulas are both graphs, while in our setting models are documents and formulas are patterns. This difference makes more complicated to apply to our framework results given in [10].

\subsection{Constraints and Clauses}

We consider three kinds of constraints: positive, negative and conditional constraints. The underlying idea of our constraints is that they should specify that certain patterns must occur (or must not occur) in a given document. For instance, the simplest kind of constraint, $\exists p$, specifies that a given document $t$ should satisfy $p$. Obviously, $\neg \exists p$ specifies that a given document $t$ should not satisfy $p$. A more complex kind of constraints is of the form $\forall(c: p \rightarrow q)$ where $c$ is a prefix function. Roughly speaking, this constraint specifies that whenever a document $t$ verifies the pattern $p$ it should also verify the extended pattern $q$ (see Definition 3.3 below). In general we will have clauses formed as disjunctions of these three types of constraints.

Definition 3.1. Given two patterns $p$ and $q$, a prefix function from $p$ to $q$ is an injective function $c$ : Nodes $(p) \rightarrow \operatorname{Nodes}(q)$ that satisfies the following conditions:

- Root-identity: $c(\operatorname{Root}(p))=\operatorname{Root}(q)$;

- Label-identity: For each $n \in \operatorname{Nodes}(p), \operatorname{Label}(n)=\operatorname{Label}(c(n))$;

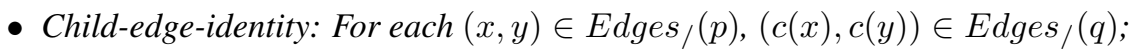

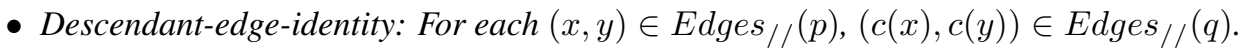

We will simply write $c: p \rightarrow q$ and we will say that $p$ is a prefix of $q$. Obviously each prefix function is a monomorphism, but the contrary is not true. See for instance that the monomorphism in Figure 3 is not a prefix function since it violates two conditions: "Label-identity" and "Descendant-edge-identity".

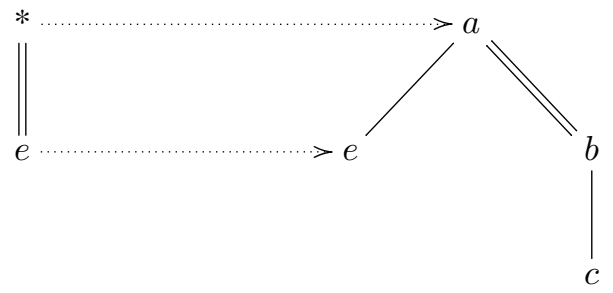

Figure 3: A monomorhism $h: p \rightarrow q$ between two patterns 
Definition 3.2. Given a pattern $p, \exists p$ denotes a positive constraint and $\neg \exists p$ denotes a negative constraint. A conditional constraint is denoted $\forall(c: p \rightarrow q)$ where $p$ and $q$ are patterns and $c: p \rightarrow q$ is $a$ prefix function.

A clause $\alpha$ is a finite disjunction of literals $L_{1} \vee L_{2} \vee \cdots \vee L_{n}$, where, for each $i \in\{1, \ldots, n\}$, the literal $L_{i}$ is a (positive, negative or conditional) constraint. The empty disjunction is called the empty clause and it can be represented by FALSE.

Satisfaction of clauses is inductively defined as follows.

Definition 3.3. A document $t \in T$ satisfies a clause $\alpha$, denoted $t \models \alpha$, if it holds:

- $t \models \exists p$ if $t \vDash p$ (that is, if there exists a monomorphism $h: p \rightarrow t$ );

- $t \models \neg \exists p$ if $t \not \models p$ (that is, if there does not exist a monomorphism $h: p \rightarrow t$ );

- $t=\forall(c: p \rightarrow q)$ if for every monomorphism $h: p \rightarrow t$ there is a monomorphism $f: q \rightarrow t$ such that $h=f \circ c$.

- $t \models L_{1} \vee L_{2} \vee \cdots \vee L_{n}$ if $t \models L_{i}$ for some $i \in\{1, \ldots, n\}$.

Let us see what satisfaction of a conditional constraint means. Consider the conditional constraint $\forall(c: p \rightarrow q)$ with $p=* / / a, q=* / / a / b$ and $c$ being the obvious prefix function from $p$ to $q$. By Definition 3.3 a document satisfies this constraint if each node (descendant of the root) labelled $a$ has a child node labelled $b$. Then the document $t=g(/ a / b)(/ a / h)$ does not satisfy the constraint. In fact, for the monomorphism $h: p \rightarrow t$ that applies the node $a$ in $p$ into the second node $a$ in $t$, there does not exist a monomorphism $f: q \rightarrow t$ such that $h=f \circ c$. However, note that $t \models q$. Therefore, in general, to verify the conditional constraint $\forall(c: p \rightarrow q)$ is stronger than to verify the clause $C=\neg \exists p \vee \exists q$.

\subsection{Specifications}

We assume that a specification consists of a set of clauses. As said in the introduction, our aim is to find a sound and complete refutation procedure for checking satisfiability of specifications consisting of clauses as defined above. Here we give an example of an unsatisfiable specification.

Example 3.1. Consider the specification $\mathcal{S}=\left\{C_{1}, C_{2}, C_{3}, C_{4}\right\}$ where $C_{1}=\exists(* / / b) \vee \exists(* / / e), C_{2}=$ $\forall\left(c_{2}: * / / b \rightarrow *(/ / b)(/ e)\right), C_{3}=\forall\left(c_{3}: * / / e \rightarrow *(/ / e)(/ b)\right)$, and $C_{4}=\neg \exists(*(/ b)(/ e))$.

Clause $C_{1}$ specifies that the document( $(s)$ must have a node labelled $b$ or $e ; C_{2}$ says that if the document has some node labelled $b$ then its root must have a child node labelled e; similarly, $C_{3}$ says that if the document has some node labelled e then the root must have a child node labelled b; and finally, $C_{4}$ says that the root cannot have two children nodes labelled $b$ and $e$. It is easy to test, for instance, that the document $t_{1}=a(/ b)(/ f / e)$ satisfies $C_{1}, C_{3}$ and $C_{4}$ but $t_{1} \not \models C_{2}$. Similarly, the document $t_{2}=a / e$ satisfies $C_{1}, C_{2}$ and $C_{4}$ but $t_{2} \not \models C_{3}$. There is no document satisfying all clauses in $\mathcal{S}$.

\section{Rules for a Refutation Procedure}

As it is often done in the area of automatic reasoning, the refutation procedure that we present in this paper is defined by means of some inference rules. Each rule tells us that if certain premises are satisfied then a given consequence will also hold. In this context, a refutation procedure can be seen as a (possibly nonterminating) nondeterministic computation where the current state is given by the set of formulas that have been inferred until the given moment, and where a computation step means adding to the given 
state the result of applying an inference rule to that state. In our case, we assume that in general the inference rules have the following form, where the premises $\Gamma_{1}, \Gamma_{2}$ and the conclusion $\Gamma_{3}$ are clauses 1 .

$$
\frac{\Gamma_{1} \quad \Gamma_{2}}{\Gamma_{3}}
$$

A refutation procedure for a set of clauses $\mathcal{S}$ is a sequence of inferences: $\mathcal{S}_{0} \Rightarrow \mathcal{S}_{1} \Rightarrow \cdots \Rightarrow \mathcal{S}_{i} \Rightarrow \ldots$ where the initial state is the original specification (i.e., $\mathcal{S}_{0}=\mathcal{S}$ ) and where we write $\mathcal{S}_{i} \Rightarrow \mathcal{S}_{i+1}$ if there is an inference rule such that $\Gamma_{1}, \Gamma_{2} \in \mathcal{S}_{i}$, and $\mathcal{S}_{i+1}=\mathcal{S}_{i} \cup\left\{\Gamma_{3}\right\}$.

In general, a refutation procedure for $\mathcal{S}$ is sound if whenever the procedure infers the empty clause it holds that $\mathcal{S}$ is unsatisfiable. And a procedure is complete if, whenever $\mathcal{S}$ is unsatisfiable, the procedure infers FALSE. In this framework, since the procedures are nondeterministic, there is the possibility that we never apply some key inference. To avoid this problem we will always assume that the procedure is fair, which means that every inference will eventually be performed (they are not postponed forever).

\subsection{Inference Rules}

Here we present three inference rules (R1), (R2) and (R3), for our refutation procedure. In our context, the clauses are disjunction of literals where each literal can be of the form $\exists p, \neg \exists p$, or $\forall(c: p \rightarrow q)$. We are going to present and explain each rule giving some examples of them.

$$
\begin{gathered}
\quad \frac{\exists p_{1} \vee \Gamma_{1} \quad \neg \exists p_{2} \vee \Gamma_{2}}{\Gamma_{1} \vee \Gamma_{2}} \\
\text { if there exists a monomorphism } m: p_{2} \rightarrow p_{1}
\end{gathered}
$$

Rule (R1) is similar to the Resolution rule, since the two premises have literals that are, in some sense, "complementary": one is a positive constraint, the other one is a negative one, and the condition about the monomorphism from $p_{2}$ to $p_{1}$ plays the same role as unification. Note that when $\Gamma_{1}$ and $\Gamma_{2}$ are empty, the rule (R1) infers the empty clause FALSE.

For instance, for $p_{1}=a(/ e)(/ / *(/ c)(/ b))$ and $p_{2}=* / / b$, there exists a monomorphism from $p_{2}$ to $p_{1}$ that applies the root of $p_{2}$ (labelled $*$ ) into the root of $p_{1}$ (labelled $a$ ), the node in $p_{2}$ labelled $b$ into the node in $p_{1}$ labelled $b$, and the edge // in $p_{2}$ into a path in $p_{1}$ formed by // followed by /. Then the empty clause $F A L S E$ is obtained as the conclusion of rule (R1) from the premises $\exists p_{1}$ and $\neg \exists p_{2}$.

$$
\frac{\exists p_{1} \vee \Gamma_{1} \quad \exists p_{2} \vee \Gamma_{2}}{\left(\bigvee_{s \in p_{1} \otimes p_{2}} \exists s\right) \vee \Gamma_{1} \vee \Gamma_{2}}
$$

Rule (R2) builds a disjunction of positive constraints from two positive constraints. It uses the operator $\otimes$ that we define below. Informally speaking, given two patterns $p_{1}$ and $p_{2}, p_{1} \otimes p_{2}$ denotes the set of patterns that can be obtained by "combining" $p_{1}$ and $p_{2}$ in all possible ways.

For instance, given the patterns $p_{1}=a(/ b / e)(/ / c)$ and $p_{2}=a / / b / x$, the set $p_{1} \otimes p_{2}$ contains the two patterns: $s_{1}=a(/ b(/ e)(/ x))(/ / c)$ and $s_{2}=a(/ b / e)(/ / b / x)(/ / c)$. Each one corresponds with a way of combining $p_{1}$ and $p_{2}$; the nodes labelled $b$ are shared in $s_{1}$ while there are two different nodes $b$ in $s 2$.

The underlying idea is that all patterns $s$ in $p_{1} \otimes p_{2}$ must verify that every document that is a model of $s$ must be a model of $p_{1}$ and a model of $p_{2}$. Conversely, every document that is a model of both $p_{1}$ and $p_{2}$ must be a model of some $s$ in $p_{1} \otimes p_{2}$. In some cases $F A L S E$ can be inferred by rule (R2).

${ }^{1}$ Clauses are seen as sets of literals, i.e. in a clause written $L \vee \Gamma, L$ is not necessarily the leftmost literal in the clause. 
Definition 4.1. Given two patterns $p_{1}$ and $p_{2}, p_{1} \otimes p_{2}$ is defined as the following set of patterns: $p_{1} \otimes p_{2}=$ $\left\{s \in P \mid\right.$ there exist jointly surjective monomorphisms inc $c_{1}: p_{1} \rightarrow s$ and $\left.i_{n} c_{2}: p_{2} \rightarrow s\right\}$ where "joinly surjective" means that Nodes $(s)=\operatorname{inc}_{1}\left(\operatorname{Nodes}\left(p_{1}\right)\right) \cup i n c_{2}\left(\operatorname{Nodes}\left(p_{2}\right)\right)$.

Lemma 4.1. Given two patterns $p_{1}$ and $p_{2}$, the set of patterns $p_{1} \otimes p_{2}$ is the empty set if and only if $p_{1}$ and $p_{2}$ have different labels in $\Sigma$. Then $\bigvee_{p \in p_{1} \otimes p_{2}} \exists p$ is the clause FALSE.

Proof. If the roots of $p_{1}$ and $p_{2}$ have different labels in $\Sigma$ (for instance, $a$ and $b$ ) then no combination $s$ is possible since $i n c_{1}: p_{1} \rightarrow s$ implies that the root of $s$ must be labelled $a$ and $i n c_{2}: p_{2} \rightarrow s$ implies that the root of $s$ must be labelled $b$. Conversely, if the roots of $p_{1}$ and $p_{2}$ have the same label $a$ (or if one of them is $a$ and the other one is $*$ ) then the document $s$ with root labelled $a$ and whose set of subtrees is the union of the subtrees of $p_{1}$ and $p_{2}$ is an element in $p_{1} \otimes p_{2}$.

$$
\frac{\exists p_{1} \vee \Gamma_{1} \quad \forall\left(c: p_{2} \rightarrow q\right) \vee \Gamma_{2}}{\left(\bigvee_{s \in p_{1} \otimes_{c, m} q} \exists s\right) \vee \Gamma_{1} \vee \Gamma_{2}}
$$

if there is a monomorphism $m: p_{2} \rightarrow p_{1}$ that cannot be extended to $f: q \rightarrow p_{1}$ such that $f \circ c=m$.

Rule (R3) is similar to (R2) in the sense that it builds a disjunction of positive constraints, but now from a positive constraint $\exists p_{1}$ and a conditional constraint $\forall\left(c: p_{2} \rightarrow q\right)$. This rule is applied when there is a monomorphism from $p_{2}$ to $p_{1}$ that cannot be extended to another one from $q$ to $p_{1}$ via $c$. That is, there is a monomorphism $m: p_{2} \rightarrow p_{1}$ but there is no monomorphism $f: q \rightarrow p_{1}$ such that $f \circ c=m$.

Rule (R3) uses the operator $\otimes_{c, m}$ that we define below. Informally speaking, given a pattern $p_{1}$, a prefix function $c: p_{2} \rightarrow q$ (that is, a pattern $q$ with prefix $p_{2}$ ) and a monomorphism $m: p_{2} \rightarrow p_{1}$, $p_{1} \otimes_{c, m} q$ denotes the set of patterns that can be obtained by combining $p_{1}$ and $q$ in all possible ways, but maintaining $p_{2}$ shared.

For instance, given the patterns: $p_{1}=a(/ b / e)(/ / c / i), p_{2}=* / / b$, and $q=*(/ / b / / a)(/ / c / d)$, with the unique possible monomorphism $m: p_{2} \rightarrow p_{1}$ and the unique possible prefix function $c: p_{2} \rightarrow q$, the set $p_{1} \otimes_{c, m} q$ contains the patterns $s_{1}=a(/ b(/ e)(/ / a))(/ / c / i)(/ / c / d)$ and $s_{2}=$ $a(/ b(/ e)(/ / a))(/ / c(/ i)(/ d))$. Note that $s_{2}$ is similar to $s_{1}$ but with only one node labelled $c$.

Definition 4.2. Given a pattern $p_{1}$, a prefix function $c: p_{2} \rightarrow q$, and a monomorphism $m: p_{2} \rightarrow p_{1}$, $p_{1} \otimes_{c, m} q$ is defined as the following set of patterns: $p_{1} \otimes_{c, m} q=\{s \in P \mid$ there exist jointly surjective monomorphisms inc $c_{1}: p_{1} \rightarrow s$ and $i_{n} c_{2}: q \rightarrow s$ such that inc $\left.c_{1} \circ m=i n c_{2} \circ c\right\}$.

The underlying idea is that all patterns $s$ in $p_{1} \otimes_{c, m} q$ must verify that every document $t$ that is a model of $s$ must be a model of $p_{1}$ and a model of $q$. However, such a document $t$ is not necessarily a model of the conditional constraint $\forall\left(c: p_{2} \rightarrow q\right)$. Conversely, every document that is a model of both $p_{1}$ and $\forall\left(c: p_{2} \rightarrow q\right)$ must be a model of some $s$ in $p_{1} \otimes_{c, m} q$, as it is established in Lemma 4.2

\subsection{Example of Refutation}

Consider the specification given in Example 3.1. $\mathcal{S}=\left\{C_{1}, C_{2}, C_{3}, C_{4}\right\}$ with $C_{1}=\exists(* / / b) \vee \exists(* / / e)$, $C_{2}=\forall\left(c_{2}: * / / b \rightarrow *(/ / b)(/ e)\right), C_{3}=\forall\left(c_{3}: * / / e \rightarrow *(/ / e)(/ b)\right)$, and $C_{4}=\neg \exists(*(/ b)(/ e))$.

We can prove that this set of clauses is unsatisfiable by applying the inference rules until obtaining the empty clause, in the following way:

1.- (R3) applied to $C_{1}$ and $C_{2}$ gives $C_{5}=\exists(*(/ / b)(/ e)) \vee \exists(* / / e)$

2.- (R3) applied to $C_{5}$ and $C_{3}$ gives $C_{6}=\exists(*(/ / b)(/ e)(/ b)) \vee \exists(*(/ e)(/ b)) \vee \exists(* / / e)$

3.- (R1) applied to $C_{6}$ and $C_{4}$ gives $C_{7}=\exists(*(/ e)(/ b)) \vee \exists(* / / e)$ 
4.- (R1) applied to $C_{7}$ and $C_{4}$ gives $C_{8}=\exists(* / / e)$

5.- (R3) applied to $C_{8}$ and $C_{3}$ gives $C_{9}=\exists(*(/ / e)(/ b))$

6.- (R3) applied to $C_{9}$ and $C_{2}$ gives $C_{10}=\exists(*(/ / e)(/ b)(/ e)) \vee \exists(*(/ b)(/ e))$

7.- (R1) applied to $C_{10}$ and $C_{4}$ gives $C_{11}=\exists(*(/ b)(/ e))$

8.- (R1) applied to $C_{11}$ and $C_{4}$ gives $F A L S E$.

It may be noted that in step 2, the disjunction $\exists(*(/ / b)(/ e)(/ b)) \vee \exists(*(/ e)(/ b))$ is the result of doing $\bigvee_{s \in p_{1} \otimes_{c, m} q} \exists s$ for $p_{1}=*(/ / b)(/ e)$ and $\forall\left(c: p_{2} \rightarrow q\right)=C_{3}$. Similarly in step 6.

\subsection{Soundness of the Inference Rules}

For proving soundness of a refutation procedure it is enough to prove the soundness of its rules. We present first a property and then prove the soundness of each rule.

\section{Proposition 4.1. (Pair Factorization Property)}

Given three patterns $p_{1}, p_{2}, r$, and two monomorphisms $f_{1}: p_{1} \rightarrow r$ and $f_{2}: p_{2} \rightarrow r$, there exists a pattern $s \in p_{1} \otimes p_{2}$, with monomorphisms inc $c_{1}: p_{1} \rightarrow s$ and $i n c_{2}: p_{2} \rightarrow s$, and there exists a monomorphism $h: s \rightarrow r$ such that $h \circ i n c_{1}=f_{1}$ and $h \circ i n c_{2}=f_{2}$. In the particular case when $r$ is a document, this property means that $r$ is a model of $s$. Graphically:

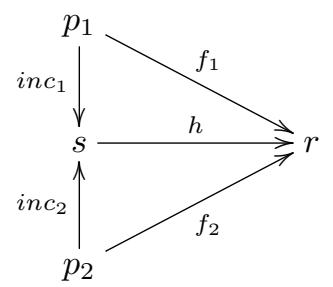

Proof. Since $f_{1}, f_{2}$ are monomorphisms, the roots of $p_{1}$ and $p_{2}$ cannot have different labels in $\Sigma$. Moreover, some $s \in p_{1} \otimes p_{2}$ holds this property. Such pattern $s$ must be chosen such that, for every $m \in \operatorname{Nodes}\left(p_{1}\right)$ and $n \in \operatorname{Nodes}\left(p_{2}\right):$ if $f_{1}(m)=f_{2}(n)$ then $i n c_{1}(m)=i n c_{2}(n)$ and if $f_{1}(m)$ is an ancestor (respectively descendant) of $f_{2}(n), i n c_{1}(m)$ must not be a descendant (respectively ancestor) of $i n c_{2}(n)$. Then $h$ is well-defined.

Lemma 4.2. Rules $(R 1),(R 2)$, and $(R 3)$ are sound.

Proof. A rule with premises $\Gamma_{1}$ and $\Gamma_{2}$ and conclusion $\Gamma_{3}$ is sound if for every document $t$ : if $t=\Gamma_{1}$ and $t \models \Gamma_{2}$ then $t \models \Gamma_{3}$.

Rule (R1). Let $t$ be a document and suppose that $t \models \exists p_{1} \vee \Gamma_{1}, t \models \neg \exists p_{2} \vee \Gamma_{2}$, and that there exists a monomorphism $m: p_{2} \rightarrow p_{1}$. It cannot happen that $t=\exists p_{1}$ and $t \models \neg \exists p_{2}$, since if $t \models \exists p_{1}$ then there exists a monomorphism $h: p_{1} \rightarrow t$ and it implies that $h \circ m: p_{2} \rightarrow t$ is also a monomorphism, meaning that $t \models \exists p_{2}$. Therefore, $t=\Gamma_{1} \vee \Gamma_{2}$.

Rule (R2). Let $t$ be a document such that $t \models \exists p_{1} \vee \Gamma_{1}$ and $t \models \exists p_{2} \vee \Gamma_{2}$. The cases where $t \models \Gamma_{1}$ or $t \models \Gamma_{2}$ are trivial. Suppose that $t \models \exists p_{1}$ and $t \models \exists p_{2}$. It means that there are two monomorphisms $h_{1}: p_{1} \rightarrow t$ and $h_{2}: p_{2} \rightarrow t$. By Proposition 4.1, there exists some $s \in p_{1} \otimes p_{2}$ verifying the pair factorization property with $h: s \rightarrow t$ being a monomorphism. Then $t \models \exists s$ and therefore $t \models \bigvee_{s \in p_{1} \otimes p_{2}} \exists s$.

Rule (R3). Let $t$ be a document such that $t=\exists p_{1} \vee \Gamma_{1}$ and $t \models \forall\left(c: p_{2} \rightarrow q\right) \vee \Gamma_{2}$, and suppose that the condition of the rule is fulfilled for the monomorphism $m: p_{2} \rightarrow p_{1}$. The cases where $t=\Gamma_{1}$ or $t \models \Gamma_{2}$ are again trivial. Suppose that $t=\exists p_{1}$ and $t \models \forall\left(c: p_{2} \rightarrow q\right)$, and let us see that $t \models \exists s$ for some $s$ in $p_{1} \otimes_{c, m} q$. Since $t=\exists p_{1}$, there exists a monomorphism $h_{1}: p_{1} \rightarrow t$. Then $h_{1} \circ m$ is also a monomorphism from $p_{2}$ to $t$. From here, since $t=\forall\left(c: p_{2} \rightarrow q\right)$, there is a monomorphism 
$h_{2}: q \rightarrow t$ such that $h_{1} \circ m=h_{2} \circ c$. On other hand, we now that for each element $s$ in $p_{1} \otimes_{c, m} q$ it holds that $i n c_{1} \circ m=i n c_{2} \circ c$. Choose $s$ such that, for each pair of nodes $x$ in $p_{1}$ and $y$ in $q$, the following properties hold:

a) If $h_{1}(x)=h_{2}(y)$ then $i n c_{1}(x)=i n c_{2}(y)$.

b) If $h_{1}(x)$ is an ancestor (respectively descendant) of $h_{2}(y)$ in $t$ then $i n c_{1}(x)$ is not a descendant (repectively an ancestor) of $i n c_{2}(y)$ in pattern $s$.

Then we can build a monomorphism $h: s \rightarrow t$ verifying $h \circ i n c_{1}=h_{1}$ and $h \circ i n c_{2}=h_{2}$. Such monomorphism $h$ is defined as follows: For each node $z$ in $i n c_{1}\left(p_{1}\right): h(z)=h_{1}\left(i n c_{1}{ }^{-1}(z)\right)$. For each node $z$ in $i n c_{2}(q): h(z)=h_{2}\left(i n c_{2}{ }^{-1}(z)\right)$. By property a), $h$ is well-defined for the nodes in $i n c_{1}\left(p_{1}\right) \cap i n c_{2}(q)$; by property b), $h: s \rightarrow t$ is a monomorphism. Therefore $t=\exists s$. Graphically:

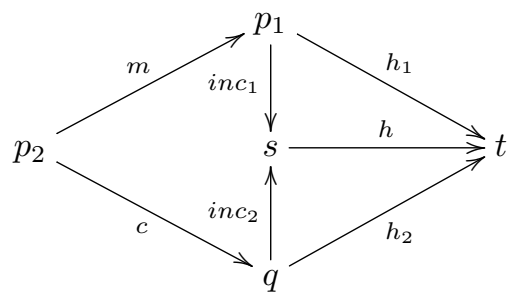

\section{Looking for Completeness}

We have seen that our refutation procedure consisting of the three inference rules (R1), (R2) and (R3) is sound, that is, whenever the procedure infers the empty clause from a set of clauses $\mathcal{S}$, we have proven that $\mathcal{S}$ is unsatisfiable. However, the procedure is not complete: It may happen that the clause FALSE is not infered by the procedure although $\mathcal{S}$ is unsatisfiable, as the following example shows.

Example 5.1. Consider the specification $\mathcal{S}=\left\{C_{1}, C_{2}, C_{3}\right\}$ which contains the clauses: $C_{1}=\exists(a / / b)$, $C_{2}=\neg \exists(a / * / / b)$, and $C_{3}=\neg \exists(a / b)$. Obviously, rules $(R 2)$ and (R3) cannot be used here. Rule $(R 1)$ cannot be applied to $C_{1}$ and $C_{2}$, because there is no monomorphism from $(a / * / / b)$ to $(a / / b)$, and $(R I)$ cannot be applied to $C_{1}$ and $C_{3}$, because there is no monomorphism from $(a / b)$ to $(a / / b)$. However $C_{1}$ is semantically equivalent to the clause $C_{1}^{\prime}=\exists(a / * / / b) \vee \exists(a / b)$, since for every document $t$ it holds: $t \models C_{1}$ if and only if $t=C_{1}^{\prime}$. Therefore $\mathcal{S}$ is unsatisfiable but our procedure does not infer FALSE.

\subsection{Unfold Rules}

The problem detected in the previous example can be solved by adding some new rules to our refutation procedure to allow unfolding a pattern like $a / / b$ in the two cases $a / b$ and $a / * / / b$. Then, by transforming $C_{1}$ into $C_{1}^{\prime}$, the procedure can infer from the set $\left\{C_{1}^{\prime}, C_{2}, C_{3}\right\}$ by applying twice the rule (R1).

As $a / * / / b$ and $a / / * / b$ are equivalent patterns, we will need to have two different ways of unfolding a descendant edge (or //-edge). The two unfolding rules are the following (to indicate a specific edge // in a tree $\mathrm{T}$ to be unfolded we will write $\mathrm{T}[/ /])$ :

\begin{tabular}{|c|cc|}
\hline $\begin{array}{c}\exists p \vee \Gamma \\
\exists p_{1} \vee \exists p_{2} \vee \Gamma\end{array} \quad$ (Unfold1) & $\exists p \vee \Gamma$ & (Unfold2) \\
for $p=T[/ /]: p_{1} \vee T[/]$ and $p_{2}=T[/, *, / /]$ & for $p=T[/ /]: p_{2} \vee \Gamma$ & $p_{1}=T / /$ and $p_{2}=T[/ /, *, /]$ \\
\hline
\end{tabular}


The rule (Unfold1) substitutes inside a clause the positive constraint $\exists p$ by $\exists p_{1} \vee \exists p_{2}$, where $p_{1}$ (respectively $p_{2}$ ) is obtained from $p$ by substituting an edge // in $p$ by the edge / in $p_{1}$ (respectively by the sequence $/, *, / /$ in $p_{2}$ ). The rule (Unfold2) is similar, but substituting // by the sequence $/ /,{ }^{*}, /$ in $p_{2}$. Both rules are sound: for every document $t$, it holds that $t=\exists p$ if and only if $t=\exists p_{1} \vee \exists p_{2}$.

With the two unfolding rules added to our refutation procedure, it is possible to infer the empty clause in more cases than without them, as we have seen in the previous example. Nevertheless, the repeated application of the unfolding rules can be infinite, giving rise to a termination problem. To avoid such a problem, the idea would be to apply the unfolding rules finitely and only in the necessary cases. We show this idea in the following example.

Example 5.2. Consider the specification $\mathcal{S}=\left\{C_{1}, C_{2}, C_{3}\right\}$ with clauses: $C_{1}=\exists(a / / c / d), C_{2}=$ $\neg \exists(a / * / d)$, and $C_{3}=\neg \exists(a / * / / * / c)$.

We can see that is not possible to apply the rule $(R 1)$ to $C_{1}$ and $C_{3}$ since there is no monomorphism from the pattern $q=(a / * / / * / c)$ to the pattern $p=a / / c / d$. However, it can be detected that a monomorphism would be possible if the edge // from a to $c$ in $p$, is unfolded until matching with the sequence $/ * / / * /$ from a to $c$ in $q$. The form of this sequence tells us to apply first (Unfold1) and then (Unfold2). More precisely: Rule (Unfold1) is applied to $C_{1}$ giving $C_{1}^{\prime}=\exists(a / c / d) \vee \exists(a / * / / c / d)$. Rule (Unfold2) is applied to $C_{1}^{\prime}$ giving $C_{1}^{\prime \prime}=\exists(a / c / d) \vee \exists(a / * / c / d) \vee \exists(a / * / / * / c / d)$. Now, the rule $(R I)$ can already be applied to $C_{1}^{\prime \prime}$ and $C_{3}$ giving $C_{4}=\exists(a / c / d) \vee \exists(a / * / c / d)$. To finish, the rule (R1) can be applied to $C_{4}$ and $C_{2}$ giving $C_{5}=\exists(a / * / c / d)$.

\subsection{Subsumption and Simplification Rules}

Finally, we can consider another classical notion, the subsumption of clauses, to build a more efficient refutation procedure. Given two clauses $C$ and $D, C$ subsumes $D$ (or equivalently $D$ is subsumed by $C$ ) if $\operatorname{Mod}(C) \subseteq \operatorname{Mod}(D)$. For instance, $\Gamma_{1}$ subsumes $\Gamma_{1} \vee \Gamma_{2}$. Subsumed clauses are redundant and it seems obvious that they must be deleted as soon as possible in the refutation procedure. However, we must have into account that, in some cases, introducing deleting rules may cause that a different strategy is needed to prove the completeness of the procedure [11]. Following with the example 5.2, we show now the subsumed clauses that can be deleted in each step of our procedure.

Example 5.3. Following the previous example we have that: $C_{1}^{\prime}$ replaces $C_{1}$ after the application of (Unfold1), therefore $C_{1}$ is deleted; $C_{1}^{\prime \prime}$ replaces $C_{1}^{\prime}$ after the application of (Unfold2), therefore $C_{1}^{\prime}$ is deleted; $C_{4}$ subsumes $C_{1}^{\prime \prime}$, so $C_{1}^{\prime \prime}$ can be deleted after the first application of $(R 1)$; and $C_{5}$ subsumes $C_{4}$, so $C_{4}$ can be deleted after the second application of (R1). Taking into account these subsumptions, the sequence of inferences from the specification $\mathcal{S}=\left\{C_{1}, C_{2}, C_{3}\right\}$ can be then summarized as follows (by applying subsumption as soon as possible):

$$
\mathcal{S}=\left\{C_{1}, C_{2}, C_{3}\right\} \Rightarrow\left\{C_{1}^{\prime}, C_{2}, C_{3}\right\} \Rightarrow\left\{C_{1}^{\prime \prime}, C_{2}, C_{3}\right\} \Rightarrow\left\{C_{4}, C_{2}, C_{3}\right\} \Rightarrow\left\{C_{5}, C_{2}, C_{3}\right\} .
$$

In this step of the refutation procedure, the set of clauses is $\{\exists(a / * / c / d), \neg \exists(a / * / d), \neg \exists(a / * / / * / c)\}$. Now no rule can be applied (note that the unfolding rules are only applied on positive constraints), therefore the procedure finishes. As FALSE has not been inferred, the actual set of clauses (and then also the initial state) is satisfiable. Moreover, the last set of clauses constitutes a new specification that is equivalent to but simpler than $\mathcal{S}$.

Here we present all subsumption and simplification rules that are used in our procedure. Apart from the general subsumption rule we mentioned before (named S1 below), we have defined other three subsumption rules (S2, S3 and S4). The meaning of such rules is that one premise disappears because it 
is subsumed by the other premise under some conditions.

$$
\frac{\Gamma_{1} \vee \Gamma_{2} \quad \Gamma_{1}}{\Gamma_{1}}
$$

$$
\frac{\exists q \vee \Gamma \quad \exists p \vee \Gamma}{\exists q \vee \Gamma}
$$

if there exists a monomorphism $m: p \rightarrow q$

$$
\frac{\neg \exists p \vee \Gamma \quad \neg \exists q \vee \Gamma}{\neg \exists p \vee \Gamma}
$$

if there exists a monomorphism $m: p \rightarrow q$

$$
\frac{\forall\left(c_{1}: x_{1} \rightarrow q_{1}\right) \vee \Gamma \quad \forall\left(c_{2}: x_{2} \rightarrow q_{2}\right) \vee \Gamma}{\forall\left(c_{1}: x_{1} \rightarrow q_{1}\right) \vee \Gamma}
$$

if there exist monomorphisms $f: x_{1} \rightarrow x_{2}$ and $g: q_{2} \rightarrow q_{1}$

Soundness of rules S2 and S3 can be proven by using Lemma 2.1 For S4 note that if $f: x_{1} \rightarrow x_{2}$ and $g: q_{2} \rightarrow q_{1}$ are monomorphisms then every model of $\forall\left(c_{1}: x_{1} \rightarrow q_{1}\right)$ is a model of $\forall\left(c_{2}: x_{2} \rightarrow q_{2}\right)$.

Other rules that are convenient for a refutation procedure are the simplification rules. We implicitly consider that the clause $\Gamma \vee L \vee L$ is the same as the clause $\Gamma \vee L$, and we add three simplification rules that correspond with the previous subsumption rules (each Sim with the corresponding $S$ ):

$$
\frac{\exists p \vee \exists q \vee \Gamma}{\exists p \vee \Gamma}
$$

(Sim2)

if there exists a monomorphism $m: p \rightarrow q$

$$
\frac{\neg \exists p \vee \neg \exists q \vee \Gamma}{\neg \exists q \vee \Gamma}
$$

(Sim3)

if there exists a monomorphism $m: p \rightarrow q$

$$
\frac{\forall\left(c_{1}: x_{1} \rightarrow q_{1}\right) \vee \forall\left(c_{2}: x_{2} \rightarrow q_{2}\right) \vee \Gamma}{\forall\left(c_{2}: x_{2} \rightarrow q_{2}\right) \vee \Gamma} \quad \text { (Sim4) }
$$

if there exist monomorphisms $f: x_{1} \rightarrow x_{2}$ and $g: q_{2} \rightarrow q_{1}$

As before, soundness of the simplification rules can be easily proved. To avoid unnecessary steps, the simplifications must be applied as soon as possible in the refutation procedure.

\subsection{Termination}

Let us study the termination of the refutation procedure: Are there examples of non-termination? Can we obtain the termination under some conditions? To answer these questions we will try to separate the different cases which we must face down.

Example 5.4. Consider the specification $\mathcal{S}=\left\{C_{1}, C_{2}, C_{3}\right\}$ with clauses $C_{1}=\exists(a / b), C_{2}=\forall\left(c_{2}\right.$ : $* / / b \rightarrow * / / b / c)$, and $C_{3}=\forall\left(c_{3}: * / / c \rightarrow * / / c / b\right)$.

$C_{2}$ says that each node labelled $b$ must have a child node labelled $c$ and $C_{3}$ says that each node labelled $c$ must have a child node labelled $b$. Since $C_{1}=\exists(a / b)$, then a document satisfying the three clauses must necessarily be a document with a infinite branch $a / b / c / b / c / \ldots$. Therefore, rule (R3) can be infinitely applied: First to $C_{1}$ and $C_{2}$ giving $C_{4}=a / b / c$, then to $C_{4}$ and $C_{3}$ giving $C_{5}=a / b / c / b$, etc. Moreover, by the subsumption rule (S2), $C_{1}$ is subsumed by $C_{4}$, and $C_{4}$ is subsumed by $C_{5}, \ldots$, and so on. Note that no more rules can be applied in this example. 
The previous example shows that "non-termination" is intrinsically linked with specifications that have only infinite models. As it could expect, the procedure will not stop for such class of specifications.

However, in the case of a satisfiable specification with a finite model, the refutation procedure will stop in a finite number of steps. This result will be obtained if two conditions hold. On one side, as commented before, the procedure must be "fair", which means that inferences are not postponed forever. For instance, suppose we add the clause $C_{4}=\neg \exists(* / / c)$ in the Example 5.4. Then, we need to apply (R1) eventually to obtain FALSE.

The second point to study is more intricate and refers to the application of the unfolding rules. These rules change a positive constraint with a //-edge into the disjunction of two positive constraints, one of which also containing a //-edge. Then obviously these rules can be infinitely applied and they can cause non-termination for a satisfiable specification. We need to control the application of the unfolding rules, using them only if necessary and in a controlled way, as viewed in Examples 5.2 and 5.3. However, to be sure that we will not need to unfold later a concrete //-edge that has already been unfolded, we must unfold it until a maximum size. The following definition help us to formalize this maximum size.

Definition 5.1. Given a pattern $p, a *$-sequence of $p$ is a sequence of the form $! * ! . . ! * !$ belonging to ( a branch of) $p$, with all its nodes (labelled *) without more children in $p$, and each! being either a /-edge or a //-edge. The length of a *-sequence is the number of nodes in the sequence. Let $L_{S}$ be the set of all *-sequences that appear either in a negative constraint $\neg \exists q$ or in the premise $p$ of a conditional constraint $\forall(c: p \rightarrow q)$ in a specification $S$. The star-length of the specification $S$ is defined as $m+1$ where $m=$ maximum of the lengths of the elements in $L_{S}$.

For instance, for the specification in the Example 5.2 the star-length is 3. This means that we do not need to apply more than 3 times the rules to unfold each //-edge (in a positive constraint). But in general we do not know a priori what is the appropriate combination of unfolding rules (we may need more than one combination), for this reason the number of times will be extended to cover all cases. In concrete, we will proceed as follows: Once we have calculated the star-length $l$ of a given specification $S$, all the $/ /$-edges in each positive constraint $\exists p$ will be unfolded by applying $l$ times the rule (Unfold 1) followed by applying $l$ times the rule (Unfold2). Indeed, these applications can be done in one summarized step.

Again in Example 5.2 $C_{1}$ is unfolded (in one step) into the clause $C=\exists(a / c / d) \vee \exists(a / * / c / d) \vee$ $\exists(a / * / * / c / d) \vee \exists(a / * / * / * / c / d) \vee \exists(a / * / * / * / * / c / d) \vee \exists(a / * / * / * / * / * / c / d) \vee \exists(a / * / *$ $/ * / / * / * / * / c / d)$. Now the rule (R1) can be repeatedly applied to $C$ and the negative constraint $C_{3}$, on the third to seventh literals in $C$, obtaining as a resovent the clause $C_{4}=\exists(a / c / d) \vee \exists(a / * / c / d)$ as we expected.

This case study can be extended to any star-length. It is worth mentioning that the definition of the star-length (Definition 5.1) also takes into account the *-sequences appearing in the premises of a conditional constraint because we may also need to unfold the //-edges in the positive constraints to see explicitly all the particular cases that may be extended by the conditional constraints by means of the rule (R3).

\section{The Refutation Procedure}

Once defined all the rules, we classify them in three groups: the first group $\mathcal{I} \mathcal{R}$ consists of the inference rules (R1), (R2) and (R3); the second group $\mathcal{S} \mathcal{R}$ consists of the subsumption rules (S1),(S2),(S3), (S4) and the simplification rules $(\operatorname{Sim} 2),(\operatorname{Sim} 3)$ and $(\operatorname{Sim} 4)$; the third group $\mathcal{U} \mathcal{R}$ consists of the unfolding rules (Unfold1) and (Unfold2).

Starting from the general definition given in Section 4 and considering now these groups of rules, we can say that a refutation procedure for a specification $\mathcal{S}$ is a sequence of states:

$$
\mathcal{S}_{0} \Rightarrow \mathcal{S}_{1} \Rightarrow \cdots \Rightarrow \mathcal{S}_{i} \Rightarrow \ldots
$$


where the initial state is the original specification (i.e., $\mathcal{S}_{0}=\mathcal{S}$ ) and where each state $\mathcal{S}_{i+1}$ is obtained from the previous state $\mathcal{S}_{i}$ by applying a rule. If this rule is in $\mathcal{I R}$, a new clause $\Gamma$ is added to $\mathcal{S}_{i}$ to obtain $\mathcal{S}_{i+1}$. Otherwise, if the rule is in $\mathcal{S} \mathcal{R}$ then $\mathcal{S}_{i+1}$ is obtained either deleting a clause in $\mathcal{S}_{i}$ (by a subsumption rule) or replacing a clause in $\mathcal{S}_{i}$ by another equivalent clause (by a simplification rule).

We can add the application of a rule in $\mathcal{U R}$ into this scheme, but we would need to keep in $\mathcal{S}_{i+1}$ both the premise and the conclusion of the rule or, alternatively, apply both unfolding rules simultanously and then replace the premise by the two conclusions. However, the application of the rules in $\mathcal{U R}$ must be controlled, therefore we will proceed as explained in the previous section:

Calculate the star-length $l$ of the specification $\mathcal{S}_{0}$ and apply the rules in $\mathcal{U R}$ in a "great summarized step", by unfolding all //-edges in each positive constraint $\exists p$ by applying $l$ times the rule (Unfold 1) followed by applying $l$ times the rule (Unfold2) and marking the remaining //-edges which are not going to be unfolded anymore. After this preprocess, we obtain a specification $\mathcal{S}^{\prime}{ }_{0}$ semantically equivalent to $\mathcal{S}_{0}$ where all necessary unfoldings (for the //-edges in the present positive constraints) are already done.

From this new specification, the algorithm proceeds by applying the rules in $\mathcal{I R}$ and $\mathcal{S R}$. This chain of states will stop within a state $\mathcal{S}_{i}$ if the clause FALSE appears in $\mathcal{S}_{i}$ or when no more rules can be applied to $\mathcal{S}_{i}$. In the former case, the procedure is finished. In the second case, $\mathcal{S}_{i}$ is the new input specification and the whole process must be repeated: apply the rules in $\mathcal{U} \mathcal{R}$ in a "great summarized step" but now only unfold the //-edges that are non-marked; $\mathcal{S}_{i}^{\prime}$ is obtained; then the algorithm will continue by applying the rules in $\mathcal{I R}$ and $\mathcal{S R}$ if such $\mathcal{S}^{\prime}{ }_{i}$ is different from $\mathcal{S}_{i}$. Otherwise, the procedure is finished. Note that, from the aplication of the rule (R3), $\mathcal{S}_{i}$ may contain new positive constraints with some //-edges marked and some //-edges non-marked.

Graphically, by using the arrow $\longrightarrow$ to express a $\mathcal{U} \mathcal{R}$ summarized step, our refutation procedure is:

$$
\mathcal{S}_{0} \longrightarrow \mathcal{S}^{\prime}{ }_{0} \Rightarrow \mathcal{S}_{1} \longrightarrow \mathcal{S}^{\prime}{ }_{1} \Rightarrow \cdots \Rightarrow \mathcal{S}_{i} \longrightarrow \mathcal{S}^{\prime}{ }_{i} \Rightarrow \mathcal{S}_{i+1} \longrightarrow \mathcal{S}^{\prime}{ }_{i+1} \Rightarrow \cdots \Rightarrow \mathcal{S}_{n} \ldots
$$

Recall that the procedure must be fair (as said in Section 5.3. A suitable implementation will also apply the rules in $\mathcal{S R}$ as soon as possible to get a better performance. Although without a formal proof yet, we claim that this procedure is sound and complete: The specification is unsatisfiable if and only if the clause FALSE is obtained in the process. Moreover, we think that the final outcome is one of the three following results:

a) If the procedure finishes with the clause $F A L S E$ in some state, then the specification is unsatisfiable.

b) If the procedure finishes and FALSE does not belong to any state, then the specification is satisfiable and it has a finite model.

c) If the procedure does not stop, then the specification is satisfiable and only has infinite models.

Finally, note that when the specification has no conditional constraints, the algorithm always finishes giving a result in cases a) or b).

\section{Conclusion and Further Work}

As said in the Introduction, our aim was to define a class of specifications on XML documents and to reason about these specifications. To this extent we have first proposed the specifications as sets of clauses, where a clause is a disjunction of constraints built on boolean XPath-patterns. In particular, we have defined three sorts of constrains: positive, negative, and conditional constraints. We have also defined when a document satisfies a constraint and therefore when a specification is satisfiable.

In order to reason about these specifications, we have studied adequate inference rules to build a sound and complete refutation procedure for checking the satisfiability of a given specification. In particular, we consider three inference rules (R1), (R2) and (R3), which take a similar format than the inference rules for graphs given in [10] but defining the appropriate operators $\left(p \otimes q\right.$ and $\left.p \otimes_{c, m} q\right)$ 
for our setting. Also some subsumption and simplification rules are added. We have proven soundness of all the rules. Then we show that some other rules are needed in order to obtain completeness. In concrete, we introduce the unfolding rules and study a way of apply them finitely. Then, assuming that the procedure is fair, the termination of the procedure rests within the own specification.

We plan to prove formally that the refutation procedure is complete; and with respect to implementation, we are building a prototype [1]. In fact, a first prototype implementing the previously described refutation procedure is available at http://www.sc.ehu.es/jiwnagom/PaginaWebLorea/ SpecSatisfiabilityTool.html, where we also explain the application's requirements. The algorithm of the refutation procedure is written in Prolog [6] but it also has a Java interface for an easy and friendly use. The main goal of this tool is to test if a given specification is satisfiable or not, showing the history of the execution. It can also be used to test if a given document is a model of a given specification and, as a subproduct, it permits to look for all the relations (monomorphisms) between two patterns or the result of the operations $p \otimes q$ and $p \otimes_{c, m} q$. The results are visually shown and therefore the tool makes these operations to be more understandable.

\section{References}

[1] Albors, J., and Navarro, M. SpecSatisfiabilityTool: A tool for testing the satisfiability of specifications on XML documents, Proceedings of PROLE 2014, to appear in eptcs (Elect. Proc. in Theoretical Computer Science).

[2] Alpuente. M., Ballis, D., and Falaschi, M. Automated Verification of Web Sites Using Partial Rewriting, Software Tools for Technology Transfer, 8 (2006), 565-585.

[3] Benedikt, M., and Koch, C. XPath Leashed, ACM Computing Surveys 41, 1 (2008).

[4] Benedikt, M., Fan, W., and Geerts, F. XPath satisfiability in the presence of DTDs. JACM 55, 2 (2008).

[5] Bidoit, N., and Colazzo D. Testing XML constraint satisfiability. Proceedings of the International Workshop on Hybrid Logic (HyLo 2006). ENTCS 174, 6 (2007), 45-61.

[6] Clocksin, W. F., Mellish, C.S. Programming in Prolog. Springer-Verlag (2003).

[7] Ellmer, E., Emmerich, W., Finkelstein, A., and Nentwich, C. Flexible Consistency Checking, ACM Transaction on Software Engineering and Methodology, 12(1) (2003), 28-63.

[8] Jelliffe, R. Schematron, Internet Document, http://xml.ascc.net/resource/ schematron/.

[9] Miklau, G., and Suciu, D. Containment and equivalence for a fragment of XPath, JACM, 51, 1 (2004).

[10] Orejas, F., Ehrig, H., and Prange, U. A Logic of Graph Constraints, Fundamental Approaches to Software Engineering, 11th Int. Conference, FASE 2008. LNCS 4961 (2008) 179-198.

[11] Pichler, R. Completeness and Redundancy in Constrained Clause Logic, LNCS 1761 (2000), 221-235.

[12] WORLD WIDE WEB CONSORTIUM. 1999a. XML path language (XPath) recommendation, http://www.w3c.org/TR/xpath/.

[13] WORLD WIDE WEB CONSORTIUM. 1999b. XSL transformations (XSLT). W3C recommendation version 1.0, http://www.w3.org/TR/xslt.

[14] WORLD WIDE WEB CONSORTIUM. 2001. XML schema part 0: Primer. W3C recommendation, http://www.w3c.org/XML/Schema.

[15] WORLD WIDE WEB CONSORTIUM. 2002. XQuery 1.0 and XPath 2.0 formal semantics. W3C working draft, http://www.w3.org/TR/query-algebra/.

[16] WORLD WIDE WEB CONSORTIUM. 2007. XML path language (XPath) 2.0. 